\title{
Efecto de la estimulación del apetito en la velocidad de recuperación ponderal de niños con desnutrición calórico-proteica
}

Drs. R. Burrows, S. M11zzo

\begin{abstract}
The effect of an appetite stimulant (Dihexazin) in the velocity of weight recovery of malnourished children was studied. Children treated with Dihexazin had greater weight increase after 1 and 2 months of treatment, than those treated with placebo. Decompensated malnourished children had greater increases only after 2 months of treatment. while compensated mainourished children increased from the first month. This study demonstrated that anorexia plays a role in the velocity of weight recovery in primary postnatal malnutrition. Appetite stimulants would shorten the time of nutritional rehabilitation.
\end{abstract}

En países en vías de desarrollo la desnutrición calórico-proteica infantil es una patología prevalente, constituyendo una causa importante de muerte de niños menores de un año. ${ }^{1}$ Esto se debe a que el desnutrido es más lábil a las infecciones, por presentar alteraciones a nivel del sistema inmunológico. ${ }^{2}$

La desnutrición infantil produce alteraciones del crecimiento y desarrollo del niño, ${ }^{3}$ afectando especialmente órganos que se encuentran en período crítico de desarrollo. ${ }^{4}$ Entre ellos al sistema nervioso, el cual puede ser dañado en grado variable de acuerdo a la intensidad, duración y época de presentación de la desnutricionn. ${ }^{5}$ Se conoce que existe un menor coeficiente intelectual y bajo rendimiento escolar en niños de países donde la desnutrición es prevalente. ${ }^{7,8,9}$ En la vida adulta este déficit intelectual inpide alcanzar el máximo de eficiencia laboral, con las consecuentes desventajas sociales y econónicas para el individuo, creando un círculo vicioso que le impide salir de la miseria y al país del subdesarrollo. ${ }^{1}$

Existen múltiples causas por las cuales un niño puede desnutrirse; entre ellas están las enfenne-

Departamento de Pediatria de la Universidad Católica de Chile e Instituto de Nutrición y Tecnología de los Alimentos, Universidad de Chile. dades crónicas caquectizantes (TBC, Ca), síndromes de malabsorción (Parasitosis, Enfermedad Celíaca), Enfermedades Sistémicas (cardiopatías, nefropatías), genetopatías y otras que producen la desnutrición secundaria. ${ }^{10}$

En países subdesarrollados la etiología más frecuente de la desnutrición calórico-proteica es la primaria, debida a un bajo aporte de nutrientes cono consecuencia de un bajo nivel socioeconómico, el cual se acompaña de muchos factores que facilitan, mantienen y agravan la desnutrición, como son infecciones a repetición, falta de estimulación psicosensorial, falta de higiene, bajo nivel cultural, mala utilización de recursos, destete precoz, alcoholismo del padre y posiblemente anorexia. ${ }^{11,12,1.3}$

En cierto porcentaje de desmutridos primarios es difícil conseguir ascensos ponderales adecuados, a pesar de una mayor oferta de alimentos, aun en ausencia de patologías agregadas. Esto indudablemente retrasa el tiempo de recuperación nutricional y expone al niño por más tiempo a su situación de desnutrido, con el consecuente mayor riesgo de un daño permanente y mayores gastos por concepto de salud.

En muchos de estos niños la anorexia es un síntoma importante y podría contribuir al mal as- 
censo ponderal. Nos pareció interesante estudiar si se acelera la recuperación nutricional al agregar al tratamiento de rehabilitación nutricional del desnutrido calórico-proteico primario un orexígeno para tratar la anorexia.

Para realizar esta experiencia se recurrió al orexigeno Dihexazin, * fármaco que actúa a nivel del centro hipotalárnico del apetito, estimulándolo a través de una disminución en la utilización periférica de la glucosa, que se manifiesta como una disminución en la diferencia arterio-venosa de glucosa. ${ }^{14}$ Este orexígeno ha sido usado en adultos con desnutrición secundaria a enfermedades caquectizantes agudas y crónicas, encontrándose significativos aumentos ponderales y del apetito. También se ha utilizado en niños anoréxicos primarios ${ }^{16} y$ secundarios, ${ }^{17}$ con buenas respuestas ponderales y orexígena. Se estudió en niños con apetito conservado, en los cuales se ha demostrado un aumento ponderal mensual mayor que el de niños no tratados.

\section{MATERIAL Y METODO}

Se realizó un ensayo de doble ciego con el orexígeno Dihexazin, ${ }^{*}$ en un grupo de 70 niños con desnutrición calórico-proteica primaria de diferentes grados, sin patología grave en el momento del estudio, que presentaban anorexia. Este grupo estaba formado por niños de ambos sexos, cuyas edades fluctuaron entre los 9 meses y los 14 años, los cuales estaban en control en los policlínicos de Nutrición, Endocrinología y Gastroenterología Infantil del Hospital Josefina Martínez de Ferrari.

Se dividieron en un grupo de 35 niños que recibieron el compuesto activo Dihexazin y otro grupo de igual número, que recibió placebo. La posología usada five de $10 \mathrm{cc}$ de jarabe, 2 veces al día por vía oral (media hora antes de las comidas? en los mayores de 2 años y 5 ec 2 veces al día en los menores de 2 años.

Se midieron en todos los niños, al ingreso, al mes y a los 2 meses de tratamiento, el peso y la talla. Se interrogó al mes de tratamiento respecto al efecto estimulante del apetito, sabor del medicamento, efectos clínicos colaterales, cumplimiento en la administración del medicamento.

\footnotetext{
*Dihexazin (Viternum) 2 metil-3 hidroxi-4 firmil 5 piridil-netil fosfato de Bis 4-(5-H Dibenzo a-d) dicloropentano 5-ilideno)-1 metil-piperidina.
}

El estado nutritivo, el grado de compensación de la desnutrición y la estructura fueron evaluados por los parametros peso para la edad, peso para la talla y talla para la edad, de acuerdo a las tablas de crecimiento pondoestatural del National Center of Health Statistics (NCHS). Se consideró eutrofia si el peso para la edad estaba entre los percentiles 25 y 75 , desnutrición de primer grado si estaba entre los percentiles 10 y 25 , desnutrición de segundo grado entre los percentiles 10 y 5 y desnutrición de tercer grado bajo el percentil 5. La desnutrición se consideró compensada si el peso para la talla estaba sobre el percentil 25 y descompensada si esta relación estaba por debajo del percentil 25. Se consideró estatura normal a una talla entre los percentiles 10 y 90 para la edad. déficit de estatura moderado entre los percentiles 5 y 10 y déficit severo bajo el percentil 5.

Las respuestas acerca del efecto estimulante del apetito, efectos colaterales, sabor del medicamento y administración fueron obtenidas a través de un cuestionario que se aplicó a las madres de los niños en estudio. Las significancias de los resultados obtenidos fueron analizadas estadisticamente a través de los test de $\mathrm{X}^{2}$, Fisher, MacNeman y T de Student.

\section{RESULTADOS}

Si bien no se tabuló la respuesta de la estatura a este medicamento, por la imposibilidad de precisar en forma segura ascensos estaturales con solo 2 meses de tratamiento, encontramos un $69 \%$ de retraso de talla en este grupo seleccionado para estudio.

El sabor del medicamento gustó en forma similar a ambos grupos, teniendo buena aceptabilidad (87\%).

Como efecto colateral presentaron somnolencia sólo 6 niños tratados con Dihexazin y 2 con placebo, ésta se presentó sólo los primeros días de tratamiento, desapareciendo posteriormente y no existiendo diferencias significativas entre ambos grupos.

La Tabla 1 muestra el ascenso porcentual de peso en el grupo total de desnutridos, después de 1 y 2 meses de tratamiento con el medicamento. Se observa que los niños tratados con Dihexazin presentan un aumento de peso significativamente mayor, tanto al mes como a los dos meses, al compararlos con el grupo control ( $p<0.0025$ ). 
TABLA 1. PORCENTAJE DE ASCENSO PONDERAL EN NINOS DESNUTRIDOS TRATADOS CON OREXIGENO.

\begin{tabular}{|c|c|c|}
\cline { 2 - 3 } & ler mes & $2{ }^{\circ}$ mes \\
\hline Dihexazin & $\begin{array}{c}305 \pm 305^{\mathrm{a}} \\
(35)^{\mathrm{b}}\end{array}$ & $\begin{array}{c}293 \pm 185 \\
(35)\end{array}$ \\
\hline Placebo & $111 \pm 200$ & $155 \pm 155$ \\
$(218)$ & $<0.0025$ \\
\hline
\end{tabular}

\footnotetext{
$a=$ Pronedio \pm 1 desviacion standard

b = NGmero de casos
}

TABLA 2. PORCENTAJE DE ASCENSO PONDERAL EN NINOS DESNUTRIDOS COMPENSADOS, TRATADOS CON UN OREXIGENO.

\begin{tabular}{|c|c|c|}
\cline { 2 - 3 } & 1 ler mes & $2{ }^{\circ}$ mes \\
\hline Dihexazin & $\begin{array}{c}380 \pm 331^{\mathrm{a}} \\
(14)^{\mathrm{b}}\end{array}$ & $\begin{array}{c}310 \pm 192 \\
(14)\end{array}$ \\
\hline Placebo & $\begin{array}{c}7 \pm 195 \\
(17)\end{array}$ & $190 \pm 136$ \\
\hline $\mathrm{P}$ & 0.005 & 0.05 \\
\hline
\end{tabular}

\footnotetext{
$a=$ promedio \pm 1 desviacion standaro

$b_{=}$nomero de casos
} 
En la Tabla 2 se muestra el ascenso porcentual de peso en el grupo de desnutridos compensados, después de uno y dos meses de tratamiento, observándose también un aumento de peso signifi- cativamente mayor en el grupo tratado con Dihexazin, tanto al mes como a los dos meses ( $p<0.005$ y $\mathrm{p}<0.05$, respectivamente).

TABLA 3. PORCENTAJE DE ASCENSO PONDERAL EN NINOS DESNUTRIDOS DESCOMPENSADOS, TRATADOS CON UN OREXIGENO.

\begin{tabular}{|c|c|c|}
\cline { 2 - 3 } & ler mes & $2{ }^{\circ} \mathrm{mes}$ \\
\hline D1hexain & $\begin{array}{c}234 \pm 292^{\mathrm{a}} \\
(19)^{b}\end{array}$ & $254 \pm 175$ \\
& $(18)$ \\
\hline Placebo & $151 \pm 210$ & $137 \pm 174$ \\
$(19)$ & $(15)$ \\
\hline p & N.S. & $<0.05$ \\
\hline
\end{tabular}

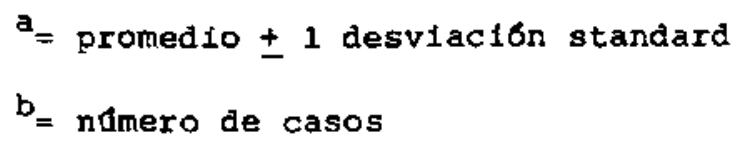

Si analizamos lo que pasa en el grupo de desnutridos (Tabla 3) descompensados, vemos que no existe diferencia significativa en el ascenso ponderal durante el primer mes de tratamiento entre el grupo tratado con el orexígeno y los tratados con placebo ( $p>0.5$ ), en cambio. esta diferencia se hace significativamente mayor para el gnupo tratado, al segundo mes de tratamiento $(\mathrm{p}<0.05$ ).

Si analizamos el estado nutritivo inicial y después de dos meses de tratamiento encontramos que los desnutridos tratados con Dihexazin mejoraron su estado mutritivo en forma significativa a los 2 meses de iniciado el tratamiento $(\mathbf{p}<0.01)$, en tanto que la mejoría del estado nutritivo a los dos meses de tratamiento, del grupo tratado con placebo, no fue significativa respecto al ingreso (p $>0.10)$ Tabla 4).

En la Tabla 5 se aprecia que existe una mejoria significativamente mayor del apetito, en el 
TAELA 4. VARIACION DEL ESTADO NUTRITIVO DE DESNUTRIDOS TRATADOS DURANTE 2 MESES CON UN OREXIGENO.

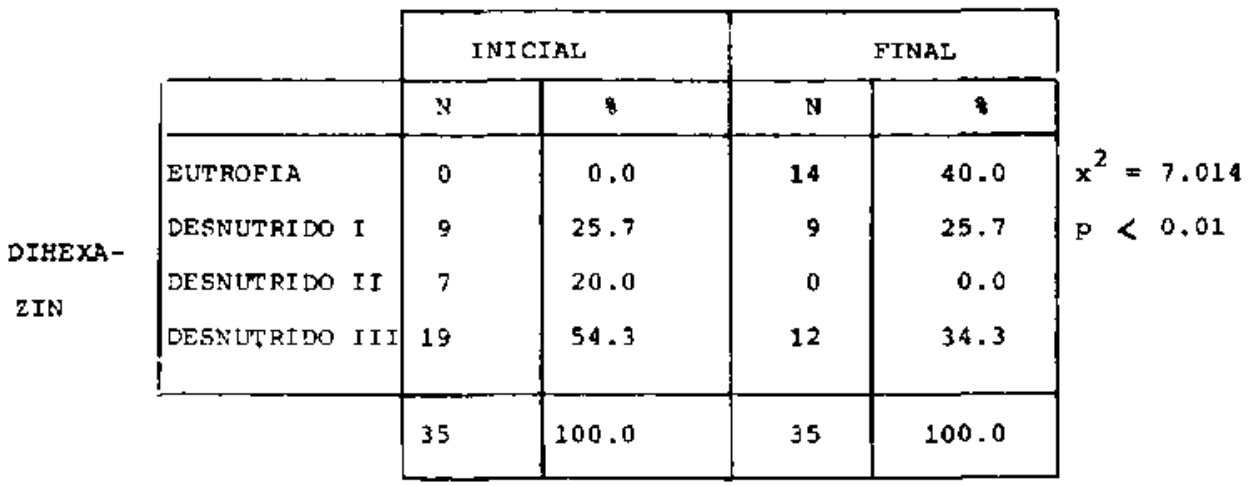

\begin{tabular}{|l|l|l|l|l|r|}
\hline PLATEOFIA & 0 & 0.0 & 6 & 21.4 \\
DESNUTRIDO I & 8 & 28.6 & 3 & 10.7 \\
DESNUTRIDO II & 6 & 21.4 & 7 & 25.0 \\
DESNUTRIDO III & 14 & 50.0 & 12 & 42.9 \\
\hline & & & & & \\
\hline
\end{tabular}

TABLA 5. PESPUESTA DEL APETITO AL TRATAMIENTO OREXIGENO EN NINOS DESNUTRIDOS.

\begin{tabular}{|l|c|c|c|c|}
\cline { 2 - 5 } \multicolumn{1}{c|}{} & \multicolumn{2}{c|}{ DIHEXAZ IN } & \multicolumn{2}{c|}{ PLACEBO } \\
\cline { 2 - 5 } & $\mathrm{N}$ & 8 & $\mathrm{~N}$ & 8 \\
\hline BUENA & 29 & 87.9 & 58 \\
\hline REGULAR & 3 & 9.0 & 5 & 17.8 \\
\hline MALA & 1 & 3.1 & 15 & 53.6 \\
\hline & 33 & 100.0 & 28 & 100.0 \\
\hline
\end{tabular}


grupo de desnutridos tratados con Dihexazin ( $p<0,0001$ ), y si separamos estos desnutridos en compensados y descompensados, encontramos que la mejoría del apetito es significativamente mayor en los tratados con el medicamento que con placebo, tanto en el grupo de desnutridos compensados como descompensados.

Esta mejoria subjetiva del apetito se produjo entre el tercer y decimoquinto día en el grupo tratado con Dihexazin, en tanto que en el grupo tratado con placebo, los pocos casos en que mejoró subjetivamente el apetito, éste varió entre el tercero y trigésimo día de tratamiento.

\section{DISCUSION}

Encontramos un significativo mayor ascenso ponderal y mejoría del apetito en los niños tratados con Dihexazin. Es interesante señalar que estos niños que presentaban una desnutrición calóricoproteica primaria, por una menor ingesta de alimentos propia de un nivel socioeconómico bajo, tuvieron una buena respuesta al medicamento, mejoraron su estado nutritivo como consecuencia de un ascenso ponderal más rápido que el grupo control. Esto indicaría, por una parte, que la anorexia sería uno de los factores que condicionarían la desnutrición y, por otro lado, que las familias de estos desnutridos, a pesar de su bajo nive] socioeconómico, serían capaces de satisfacer la mayor demanda de alimentos secundaria a un mejor apetito. Por lo tanto, el uso de un orexígeno sería de utilidad en aquellos desnutridos en los que la anorexia está presente.

Es interesante señalar que en el grupo de desnutridos descompensados, aun cuando respondieron rápidamente con un incremento del apetito al tratamiento orexígeno, sin embargo no presentaron una significativa mayor respuesta ponderal el primer mes de tratamiento. Este fenómeno podria explicarse por la presencia de alteraciones metabólicas propias del desnutrido descompensado, que le impidieron una respuesta ponderal rápida durante el primer mes, alteraciones que se habrian normalizado en el segundo mes de tratamiento, permitiéndole un significativo aumento ponderal durante este segundo período de tratamiento.

En conclusión, este estudio demuestra que la anorexia jugaría un rol en la velocidad de recupe- ración nutricional del desnutrido primario. El uso de un orexígeno, al acelerar la recuperación nutricional, signiticaría una curación más rápida y por ende una economía en el tratamiento de estos pacientes, especialmente en el caso de nuestro país, donde un importante porcentaje del presupuesto nacional se utiliza para prevenir $y$ tratar la desmutrición infantil aguda y sus consecuencias.

\section{RESUMEN}

Se estudió el efecto del orexigeno Dihexazin (Viternum M.R.) en la velocidad de recuperación ponderal de niños con desnutrición calorico-proteica primaria.

Los niños tratados con Dihexazin subieron significativamente más de peso al $10^{\circ}$ y $20^{\circ}$ mes de tratamiento que los niños tratados con placebo. Los desnutridos descompensados subieron sólo al segundo mes, mientras que los compensados subieron en forma significativa desde el primer mes.

Este estudio demostró que la anorexia sería uno de los factores que juegan un papel en la velocidad de recuperación ponderal del desnutrido primario. Por lo tanto, el uso de orexígeno acortarfa el período de recuperación nutricional.

\section{REFERENCIAS}

\footnotetext{
lMonckeberg, F. La desnutrictón en el niño y sus consecuencias.Cuad. Educ: Serie Orientaciones 5: 2, 1973.

${ }^{2}$ Chandra, H. K. (1976). Immunalogical consequences of malnutrition including fetal growth retardation.In: Food and Immunology (Symposio of the Swedish Nutrition Foundation XIII) Edited by L. Hambreans. Almqvist and Wikselle International, Stockholm.

${ }^{3}$ Monckeberg, F. La desnutricion en el nino y sus consecuencias, En: Muzzo, S.; Ballester, D., eds. Fundamentos de Nurrición. Santiago, Depto. de Nutrición y Tecnología de los Alimentos, $\mathrm{C}$, de Chile, 1975 (Publicación Dacente N. 4). 2: pp. 171-209.

4Winick, M.; Noble, A. Cuantitatives changes in DNA, RNA and Protein during prenatal and postnatal growth in the rat Devel. Biol. 12: 451, 1965.

5inick, M.; Noble, A. Cellular response in rats during mal. mutrition at various age. J. Nutrition 89: 300, 1966.

${ }^{6}$ Rozocsky, J.; Novoa, F.; Abarzu, J.; Monckeberg, F. Cranial transillurnination in early and severe malnutrition. Brit. J. Nutr. 25: 107, 1971.
} 


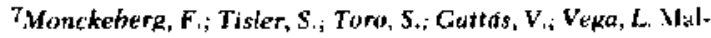
nutrition itrd mental developinent. Am. J. Clin. Nutr. 25: 766, 1972.

"Harner, R.H. Dual role of environneutal deprivution and malnutrition in retarding intellectual development. Am. J. Clin. Nitte. 29: $912,1976$.

Monckeberg. $F$. Hialuntrition and mental capacily. En: Pallitnerican Health Orgauization. Nutritimn, the Vervolss System and Behayior. Washington. PAHO. 1972 iScientific Publication .N." 251), pp. 48-54.

111Lacassie. Y.; Colombo, M.; Lopez, I. Desnutrición secundaria:

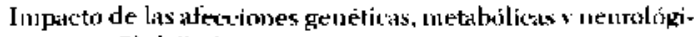
cas. Rev: Chil. Ped. 51: 257, 193 .

Ll Janja, S, . Ifartnet, J, Apra, J, L.; Acuña, A. R. Alguutos aspectos etiologicos de la desmutrición infantil en el Area Oriente de Santiago, Rev. Chil. Pediatr. 40: 85, 1969.

12Monckeberg, F. Factors conditiming malmutrition in Latin American with spectial reference to Chile. Advices tor a solum- teers action. En: Gyorky, P.; Kline, O. L. Malutrition is a problem of Ecology. Batsel, S. Kurgir. pp. 23-33.

13 Monckeberg, F. Factores que condicionan la destutricion y posibles suluctones. En; Muszo, S.; Ballester, D., eds. Fundamentes de Vutricion. Santiako. Depto. de Nutricion y Tecno logía de los Alimentos. Li te Chile, 1975 (Publicución Docente N.\% 4). 2; pp. 157-170.

HDocumentación Farmacologica de Dihexazin entiodo al SNS para aprobar uso de este fámaco.

15 Toronjo, J. Hesultado estadistico de ul ensayo slinico con un nuevo estimulante de] apetito: Vitemum. Med. Klin. [42: I35. 1973 .

16 Echeverra, j. M. Ensayo clínico del compuestu Cl-51 cono estimbulante del apetíto en un gnupo de 30 ninos. Panminerva Médica. 10: 318, 1972.

I'Da Groca, A. M. Ensaio slinico duplamente cego dum novo estimulaute do apetite, Dihexazin, num grupo de 36 criancas com deficiencias motorss. Rev. Port. Ped. 6: 270. 1975. 